Math. Model. Nat. Phenom.

Vol. 5, No. 4, 2010, pp. 269-292

DOI: $10.1051 / \mathrm{mmnp} / 20105412$

\title{
The Algebraic Multiplicity of Eigenvalues and the Evans Function Revisited
}

\author{
Y. Latushkin ${ }^{1}$ and A. Sukhtayev \\ Department of Mathematics, University of Missouri, Columbia, MO 65211, USA
}

Dedicated to the memory of M. S. Birman

\begin{abstract}
This paper is related to the spectral stability of traveling wave solutions of partial differential equations. In the first part of the paper we use the Gohberg-Rouche Theorem to prove equality of the algebraic multiplicity of an isolated eigenvalue of an abstract operator on a Hilbert space, and the algebraic multiplicity of the eigenvalue of the corresponding Birman-Schwinger type operator pencil. In the second part of the paper we apply this result to discuss three particular classes of problems: the Schrödinger operator, the operator obtained by linearizing a degenerate system of reaction diffusion equations about a pulse, and a general high order differential operator. We study relations between the algebraic multiplicity of an isolated eigenvalue for the respective operators, and the order of the eigenvalue as the zero of the Evans function for the corresponding first order system.
\end{abstract}

Key words: Fredholm determinants, non-self-adjoint operators, Evans function, linear stability, traveling waves

AMS subject classification: 35P05, 47A55, 47E05

\footnotetext{
${ }^{1}$ Corresponding author. E-mail: latushkiny@missouri.edu
} 


\section{Introduction}

In this paper we continue the work began in $[5,6,7]$, and investigate further the connections of the Evans function and (modified) Fredholm determinants of the Birman-Schwinger type operators. In particular, we bring into the discussion a new element, the Gohberg-Rouche Theorem [9, Theorem XI.9.1]. Also, we study in detail three important concrete cases: the Schrödinger operator, the operator obtained by linearizing a system of degenerate reaction diffusion equations about a traveling wave, and a general high order differential operator.

In Section 2, we deal with abstract perturbations. We first recall well-known results from [9, Ch. XI] regarding the algebraic multiplicity $m\left(\lambda_{0} ; W(\cdot)\right)$ of an isolated eigenvalue $\lambda_{0}$ of finite type for an operator pencil $W=W(\lambda)$. Next, following [6], we consider a class of factorable non-selfadjoint perturbations, formally given by $B^{*} A$, of a given unperturbed non-self-adjoint operator $H_{0}$ in a Hilbert space by introducing a densely defined, closed linear operator $H$ which represents an extension of $H_{0}+B^{*} A$. Furthermore, we discribe the properties of the Birman-Schwinger type operator pencil $K=K(\lambda)$ associated with $H_{0}$ and $H$ by the formula $K(\lambda)=-\overline{A\left(H_{0}-\lambda\right)^{-1} B^{*}}$. Under appropriate assumptions (including that $\lambda_{0}$ is an isolated eigenvalue of $H$ of finite algebraic multiplicity denoted by $m\left(\lambda_{0} ; H\right)$ ), and using the Gohberg-Rouche Theorem, we show the equality $m\left(\lambda_{0} ; I-K(\cdot)\right)=m\left(\lambda_{0} ; H\right)$ (see Theorem 18). In turn, this leads to the fact that $m\left(\lambda_{0} ; H\right)$ is the order of the zero at $\lambda_{0}$ for the modified Fredholm determinant, that is, $\operatorname{det}_{2}(I-K(\lambda))=$ $\left(\lambda-\lambda_{0}\right)^{m\left(\lambda_{0} ; H\right)} S(\lambda), S\left(\lambda_{0}\right) \neq 0$, (see Theorem 21). We mention [10,11] where yet another application of the Gohberg-Rouche Theorem is given.

In Section 3 we discuss three particular classes of problems: the Schrödinger equation, the degenerate reaction-diffusion system of equations, and a general higher order differential operator with constant leading coefficient. The main tool in our investigation is the connection of the (modified) Fredholm determinant of the Birman-Schwinger type integral operator for the linearized eigenvalue problem of a given partial differential equation, and the Evans function for the equivalent to this eigenvalue problem first order system, see $[5,12,13]$. Our strategy can be described as follows. Consider the (higher order, space dimension one) differential operator $H$ obtained by linearizing a partial differential equation along a steady state or traveling wave solution. The operator $H$ is a perturbation of the operator $H_{0}$ determined by the asymptotic behavior of the solution. As in Section 2, we associate to $H$ and $H_{0}$ a Birman-Schwinger type operator pencil $I-K(\cdot)$. To pass to the Evans function analysis, we re-write the eigenvalue problem $H u=\lambda u$ for $H$ as a first order system of differential equations $d y / d x=\mathbb{M}(x, \lambda) y(x), x \in \mathbb{R}$, and consider its Evans function $\mathbb{E}=\mathbb{E}(\lambda)$, see $[1,4,15,18,20]$. Also, we consider the corresponding first order differential operator $T(\lambda)=\partial_{x}-\mathbb{M}(x, \lambda)$. The operator $T(\lambda)$ is a perturbation of the first order differential operator $T_{0}(\lambda)$ obtained from the eigenvalue problem $H_{0} u=\lambda u$ for $H_{0}$. We associate to $T(\lambda)$ and $T_{0}(\lambda)$ a Birman-Schwinger type operator pencil $I-\mathbb{K}(\lambda)$. For the three classes of problems considered in Section 3 we show that the (modified) Fredholm determinants for $I-K(\lambda)$ and $I-\mathbb{K}(\lambda)$ are equal (see Lemma 25, (3.48) and (3.65)). Now the abstract results from Section 2 imply that the algebraic multiplicity $m\left(\lambda_{0} ; H\right)$ of a discrete eigenvalue $\lambda_{0}$ of $H$ coincides with the multiplicity of the zero at $\lambda_{0}$ for the function $\operatorname{det}_{2}(I-\mathbb{K}(\cdot))$. We recall that the main result in [5] is an explicit formula relating $\operatorname{det}_{2}(I-\mathbb{K}(\cdot))$ and the Evans function $\mathbb{E}=\mathbb{E}(\lambda)$ for the equation 
$d y / d x=\mathbb{M}(x, \lambda) y(x)$. This leads to the equalities

$$
\operatorname{det}_{2}(I-K(\lambda))=\operatorname{det}_{2}(I-\mathbb{K}(\lambda))=e^{\Theta(\lambda)} \mathbb{E}(\lambda)=\left(\lambda-\lambda_{0}\right)^{m\left(\lambda_{0} ; H\right)} S(\lambda),
$$

where $S\left(\lambda_{0}\right) \neq 0$, and $\Theta(\lambda)$ is a function, analytic in $\lambda$, explicitly computed in [5].

In particular, (1.1) shows that the algebraic multiplicity $m\left(\lambda_{0} ; H\right)$ is equal to the multiplicity of the zero at $\lambda_{0}$ of the Evans function. The latter assertion is well-known and proved in many concrete situations, see, e.g., $[1,4,18,15,20]$ and the literature therein. To conclude this introduction, we will briefly review the main insight in the classical strategy of the proof of this assertion as it is quite different from ours. First, one remarks that $\lambda_{0}$ is an eigenvalue of $H$ if and only if 0 is an eigenvalue of $T\left(\lambda_{0}\right)$. However, unlike $H$, the operator $T\left(\lambda_{0}\right)$ does not have isolated eigenvalues (in fact, it is easy to see that the spectrum of $T\left(\lambda_{0}\right)$ is invariant with respect to vertical translations, cf. [3, Prop. 2.36(b)]). Thus, the "algebraic multiplicity" of 0 as an eigenvalue of $T\left(\lambda_{0}\right)$ is defined via the lengths of the Jordan chains. Namely, since the higher order differential equation $\left(H-\lambda_{0}\right) u=0$ generates the first order differential equation $d y / d x=\mathbb{M}\left(x, \lambda_{0}\right) y(x)$, one observes that a Jordan chain $\left\{u_{j}\right\}_{j=1}^{\ell}$ for $\lambda_{0}$, satisfying $u_{j-1}=\left(H-\lambda_{0}\right) u_{j}, u_{0}=0, j=1, \ldots, \ell$, generates the chain of functions $\left\{y_{j}\right\}_{j=1}^{\ell}$, satisfying $T\left(\lambda_{0}\right) y_{j}=\mathbb{M}^{\bullet}\left(\cdot, \lambda_{0}\right) y_{j-1}, j=1, \ldots, \ell, y_{0}=0$. Here, ${ }^{\bullet}$ denotes differentiation in $\lambda$. Differentiating in $\lambda$ the first order differential equation $d y / d x=\mathbb{M}\left(x, \lambda_{0}\right) y(x)$, we arrive at the equation $d y^{\bullet} / d x=\mathbb{M}\left(x, \lambda_{0}\right) y^{\bullet}(x)+\mathbb{M}^{\bullet}\left(x, \lambda_{0}\right) y$ which, in fact, is very close to the equation for the Jordan chain. Using this main observation, the equality of the algebraic multiplicity of $\lambda_{0}$ and the multiplicity as the zero of the Evans function follows using some elementary but extremely clever computations with the derivative of the latter, see, e.g., $[1,4,18,15,20]$ and the literature therein.

In this paper, we use the following notation. Let $\mathcal{H}$ and $\mathcal{K}$ be separable complex Hilbert spaces, $(\cdot, \cdot)_{\mathcal{H}}$ and $(\cdot, \cdot)_{\mathcal{K}}$ the scalar products in $\mathcal{H}$ and $\mathcal{K}$ (linear in the second factor), and $I_{\mathcal{H}}$ and $I_{\mathcal{K}}$ the identity operators in $\mathcal{H}$ and $\mathcal{K}$, respectively. Next, let $T$ be a closed linear operator from $\operatorname{dom}(T) \subseteq \mathcal{H}$ to $\operatorname{ran}(T) \subseteq \mathcal{K}$, with $\operatorname{dom}(T)$ and $\operatorname{ran}(T)$ denoting the domain and range of $T$. The closure of a closable operator $S$ is denoted by $\bar{S}$. The kernel (null space) of $T$ is denoted by $\operatorname{ker}(T)$. The spectrum and resolvent set of a closed linear operator in $\mathcal{H}$ will be denoted by $\sigma(\cdot)$ and $\rho(\cdot)$. The Banach spaces of bounded and compact linear operators in $\mathcal{H}$ are denoted by $\mathcal{B}(\mathcal{H})$ and $\mathcal{B}_{\infty}(\mathcal{H})$, respectively. Similarly, the Schatten-von Neumann (trace) ideals will subsequently be denoted by $\mathcal{B}_{p}=\mathcal{B}_{p}(\mathcal{H}), p \in \mathbb{N}$. Analogous notation $\mathcal{B}(\mathcal{H}, \mathcal{K}), \mathcal{B}_{\infty}(\mathcal{H}, \mathcal{K})$, etc., will be used for bounded, compact, etc., operators between two Hilbert spaces $\mathcal{H}$ and $\mathcal{K}$. In addition, $\operatorname{tr}(T)$ denotes the trace of a trace class operator $T \in \mathcal{B}_{1}(\mathcal{H})$ and $\operatorname{det}_{p}\left(I_{\mathcal{H}}+S\right)$ represents the (modified) Fredholm determinant associated with an operator $S \in \mathcal{B}_{p}(\mathcal{H}), p \in \mathbb{N}$, see [19]. For a closed operator $T$ we denote by $\left(\operatorname{dom}(T),\|\cdot\|_{T}\right)$ its domain equipped with the graph norm $\|f\|_{T}=\left(\|f\|^{2}+\|T f\|^{2}\right)^{1 / 2}$. We denote by $L^{2}(\mathbb{R} ; d x)^{n}$ and $L^{2}(\mathbb{R} ; d x)^{n \times n}$ the space of $(n \times 1)$ vector valued functions and $(n \times n)$ matrix valued functions, respectively. 


\section{Abstract Perturbation Theory}

To make the exposition self-contained, we begin by reminding some known facts from [9, Chap. $\mathrm{XI}]$. Let $W: \Omega \rightarrow \mathcal{B}(\mathcal{H})$ be an operator-valued function analytic in an open domain $\Omega$ of the complex plane. Assume that the operator $W\left(\lambda_{0}\right)$ is Fredholm of index zero for some $\lambda_{0} \in \Omega$. Then there exits an operator $F: \mathcal{H} \rightarrow \mathcal{H}$ of finite rank such that $W\left(\lambda_{0}\right)+F$ is invertible. Since $W(\lambda)$ is continuous in $\lambda$, the operator $E(\lambda)=W(\lambda)+F$ is invertible for $\lambda$ in some open disc $\left|\lambda-\lambda_{0}\right|<\delta_{0}$, and thus

$$
W(\lambda)=E(\lambda)-F=E(\lambda)\left[I-E(\lambda)^{-1} F\right], \quad\left|\lambda-\lambda_{0}\right|<\delta_{0} .
$$

Since $F$ is the operator of finite rank, ker $F$ has a finite dimensional complement $\mathcal{H}_{0}$ in $\mathcal{H}$. Let $P$ be the projection of $\mathcal{H}$ along ker $F$ onto $\mathcal{H}_{0}$. It follows that

$$
I-E(\lambda)^{-1} F=\left[I-P E(\lambda)^{-1} F P\right]\left[I-(I-P) E(\lambda)^{-1} F P\right] .
$$

We put $G(\lambda)=I-(I-P) E(\lambda)^{-1} F P$ and note that $G$ is well-defined and analytic in the disc $\left|\lambda-\lambda_{0}\right|<\delta_{0}$. Furthermore, $G$ takes values in the set of invertible operators on $\mathcal{H}$; in fact

$$
G(\lambda)^{-1}=I+(I-P) E(\lambda)^{-1} F P,\left|\lambda-\lambda_{0}\right|<\delta_{0} .
$$

Combining this together, we infer:

$$
W(\lambda)=E(\lambda)\left[I-P E(\lambda)^{-1} F P\right] G(\lambda), \quad\left|\lambda-\lambda_{0}\right|<\delta_{0},
$$

where $E$ and $G$ are analytic operator-valued functions on $\left|\lambda-\lambda_{0}\right|<\delta_{0}$ and their values are invertible operators.

Definition 1. Let $\Omega$ be an open set in $\mathbb{C}$, and let $T(\cdot)$ and $S(\cdot)$ be operator-valued functions defined on $\Omega$. Given $\lambda_{0}$ in $\Omega$, we say that $T(\cdot)$ and $S(\cdot)$ are equivalent at $\lambda_{0}$ if there exists an open neighborhood $\mathcal{U}$ of $\lambda_{0}$ in $\Omega$ such that

$$
T(\lambda)=F(\lambda) S(\lambda) E(\lambda), \quad \lambda \in \mathcal{U},
$$

where $F(\cdot)$ and $E(\cdot)$ are invertible operators which depend analytically on $\lambda$ in $\mathcal{U}$.

We isolate a part of the proof of Theorem XI.8.1 in [9] as the following lemma.

Lemma 2. [9, pp. 200-201] Assume that

$$
W_{0}(\lambda)=\left[a_{i j}(\lambda)\right]_{i, j=1}^{n},
$$

where $a_{i j}$ are scalar-valued functions that are analytic at $\lambda_{0}$. Then $W_{0}(\cdot)$ is equivalent at $\lambda_{0}$ to an analytic operator-valued function $D_{0}$ of the form

$$
D_{0}(\lambda)=\pi_{0}+\left(\lambda-\lambda_{0}\right)^{k_{1}} \pi_{1}+\ldots+\left(\lambda-\lambda_{0}\right)^{k_{r}} \pi_{r}, \quad k_{1} \leq k_{2} \ldots \leq k_{r},
$$

where $\pi_{0}, \pi_{1}, \ldots, \pi_{r}$ are mutually disjoint projections in $\mathbb{C}^{n}$ such that rank $\pi_{j}=1$ for $j=1, \ldots, r$. 
Proof. If all entries $a_{i j}$ are identically zero in a neighborhood of $\lambda_{0}$, then the theorem is trivially true. Therefore, assume that for at least one pair $(i, j)$ the function $a_{i j}$ does not vanish identically in a neighborhood of $\lambda_{0}$. In that case we may write

$$
a_{i j}(\lambda)=\left(\lambda-\lambda_{0}\right)^{l(i, j)} b_{i j}(\lambda),
$$

where $b_{i j}\left(\lambda_{0}\right) \neq 0$ and $l(i, j) \in \mathbb{N} \cup\{0\}$. Choose $\left(i_{0}, j_{0}\right)$ in such a way that the number $l\left(i_{0}, j_{0}\right)$ is minimal. By renumbering rows and columns in (2.5) we may assume without loss of generality that $i_{0}=1, j_{0}=1$. Furthermore, by multiplying $W_{0}(\lambda)$ on the left by the diagonal matrix $E(\lambda)=$ $\operatorname{diag}\left[b_{11}(\lambda)^{-1}, 1, \ldots, 1\right]$, we may suppose that $a_{11}=\left(\lambda-\lambda_{0}\right)^{k_{1}}$ and $a_{i j}=\left(\lambda-\lambda_{0}\right)^{k_{1}} c_{i j}(\lambda)$, where $c_{i j}$ is analytic at $\lambda_{0}$. Note that the diagonal matrix $E(\lambda)$ is invertible and $E(\lambda)$ depends analytically on $\lambda$ in a neighborhood of $\lambda_{0}$. Thus multiplication by $E(\lambda)$ produces an equivalent at $\lambda_{0}$ matrixvalued function $E(\lambda) W_{0}(\lambda)$.

Next, in the matrix $E(\lambda) W_{0}(\lambda)$ we subtract $c_{i 1}$ times the first row from the $i$-th row, that is, multiply $E(\lambda) W_{0}(\lambda)$ from the left by an elementary matrix which is invertible and depends analytically on $\lambda$ in a neighborhood of $\lambda_{0}$. In the resulting product, we subtract $c_{1 j}$ times the first column from the $j$-th column, and we will do this for $1 \leqslant i, j \leqslant n$. It follows that $W_{0}$ is equivalent at $\lambda_{0}$ to an operator function of the form

$$
\left[\begin{array}{cccc}
\left(\lambda-\lambda_{0}\right)^{k} & 0 & \cdots & 0 \\
0 & \alpha_{22}(\lambda) & \cdots & \alpha_{2 n}(\lambda) \\
\vdots & \vdots & & \vdots \\
0 & \alpha_{n 2}(\lambda) & \cdots & \alpha_{n n}(\lambda)
\end{array}\right]
$$

where $\alpha_{i j}(\lambda)=\left(\lambda-\lambda_{0}\right)^{k_{1}} \beta_{i j}(\lambda)$ with $\beta_{i j}$ analytic at $\lambda_{0}$. By applying induction by the dimension of the submatrix, the lemma is proved.

Remark 3. The procedure, described in the proof of Lemma 2, will either (a) exhaust the dimension of the remain submatrix, or $(b)$ the remaining submatrix will become identically zero in a neighborhood of $\lambda_{0}$. In case $(a), \pi_{0}+\pi_{1}+\ldots+\pi_{r}=I$.

Theorem 4. [9, Theorem XI.8.1] Let $W: \Omega \rightarrow \mathcal{B}(\mathcal{H})$ be an analytic operator-valued function, and assume that for some $\lambda_{0} \in \Omega$ the operator $W\left(\lambda_{0}\right)$ is Fredholm with index zero. Then $W$ is equivalent at $\lambda_{0}$ to an analytic operator-valued function $D$ of the form

$$
D(\lambda)=P_{0}+\left(\lambda-\lambda_{0}\right)^{k_{1}} P_{1}+\ldots+\left(\lambda-\lambda_{0}\right)^{k_{r}} P_{r},
$$

where $P_{0}, P_{1}, \ldots, P_{r}$ are mutually disjoint projections such that $P_{1}, \ldots, P_{r}$ have rank one, the projection $I-P_{0}$ has finite rank, and $k_{1} \leq k_{2} \ldots \leq k_{r}$. Moreover, there exist operators $E(\lambda), G(\lambda)$ on $\mathcal{H}$ and $G_{0}$ on $\mathcal{H}_{0}$ so that

$$
W(\lambda)=E(\lambda) G_{1}(\lambda) D(\lambda) G(\lambda),
$$

where $G_{1}(\lambda)=\left[\begin{array}{cc}G_{0}(\lambda) & 0 \\ 0 & I_{\operatorname{ker} P}\end{array}\right]: \operatorname{ran} P \oplus \operatorname{ker} P \rightarrow \operatorname{ran} P \oplus \operatorname{ker} P$. In addition, $E(\cdot), G_{1}(\cdot), G(\cdot)$ are analytic and take invertible values. 
Proof. According to formula (2.3) the operator-valued function $W$ is equivalent at $\lambda_{0}$ to an operator function of the form

$$
\left[\begin{array}{cc}
W_{0}(\cdot) & 0 \\
0 & I_{\text {ker } P}
\end{array}\right]: \operatorname{ran} P \oplus \operatorname{ker} P \rightarrow \operatorname{ran} P \oplus \operatorname{ker} P
$$

Here $W_{0}(\cdot)$ is holomorphic on $\left|\lambda-\lambda_{0}\right|<\delta_{0}$ and $W_{0}(\lambda)$ acts on the finite dimensional space $\mathcal{H}_{0}=\operatorname{ran} P$. By Lemma $2 W_{0}$ is equivalent at $\lambda_{0}$ to an operator-valued function $D_{0}$ of the form

$$
D_{0}(\lambda)=\pi_{0}+\left(\lambda-\lambda_{0}\right)^{k_{1}} \pi_{1}+\ldots+\left(\lambda-\lambda_{0}\right)^{k_{r}} \pi_{r}
$$

where $\pi_{0}, \pi_{1}, \ldots, \pi_{r}$ are mutually disjoint projections on $\operatorname{ran} P$ and $\operatorname{rank} \pi_{j}=1$ for $j=1, \ldots, r$. Put $P_{j}=\pi_{j} P$ for $j=1, \ldots, r$, and let

$$
P_{0}=\left[\begin{array}{cc}
\pi_{0} & 0 \\
0 & I_{\text {ker } P}
\end{array}\right]: \operatorname{ran} P \oplus \operatorname{ker} P \rightarrow \operatorname{ran} P \oplus \operatorname{ker} P .
$$

Then the operator function (2.10) (and hence $W$ ) is equivalent at $\lambda_{0}$ to the function

$$
D(\lambda)=P_{0}+\left(\lambda-\lambda_{0}\right)^{k_{1}} P_{1}+\ldots+\left(\lambda-\lambda_{0}\right)^{k_{r}} P_{r},
$$

and $P_{0}, P_{1}, \ldots, P_{r}$ have the desired properties.

Corollary 5. The operators $\left(G_{1}(\lambda)-I\right),(D(\lambda)-I),(G(\lambda)-I)$, see (2.9), are of finite rank and, therefore, belong to $\mathcal{B}_{p}$ for every $p$.

Definition 6. We say that $\lambda_{0} \in \Omega$ is an eigenvalue of finite type of an analytic function $W: \Omega \rightarrow$ $\mathcal{B}(\mathcal{H})$ if $W\left(\lambda_{0}\right)$ if Fredholm, $W\left(\lambda_{0}\right) f=0$ for some non-zero $f \in \mathcal{H}$, and $W(\lambda)$ is invertible for all $\lambda$ in some punctured disc $0<\left|\lambda-\lambda_{0}\right|<\varepsilon$ around $\lambda_{0}$.

If $\lambda_{0}$ is an eigenvalue of finite type, then ind $W\left(\lambda_{0}\right)=0$, and hence, by Theorem 4, the operator-valued function $W$ is equivalent at $\lambda_{0}$ to the operator-valued function of the form

$$
D(\lambda)=P_{0}+\left(\lambda-\lambda_{0}\right)^{k_{1}} P_{1}+\ldots+\left(\lambda-\lambda_{0}\right)^{k_{r}} P_{r},
$$

where $P_{0}, P_{1}, \ldots, P_{r}$ are as in Theorem 4 and satisfy the additional condition

$$
P_{0}+P_{1}+\ldots+P_{r}=I
$$

which follows from the fact that $D(\lambda)$ is invertible for $\lambda \neq \lambda_{0}$ and $\lambda$ sufficiently close to $\lambda_{0}$.

Definition 7. The sum $k_{1}+k_{2}+\ldots+k_{r}$ of the indices in (2.14) is called the algebraic multiplicity of $W$ at $\lambda_{0}$, and is denoted by $m\left(\lambda_{0} ; W(\cdot)\right)$.

The following result is called the Gohberg-Rouche Theorem. 
Theorem 8. [9, Theorem XI.9.1] Let $W: \Omega \rightarrow \mathcal{B}(\mathcal{H})$ be an analytic operator-valued function and $\lambda_{0}$ be an eigenvalue of finite type of $W(\cdot)$. Then, there is a Cauchy contour $\Gamma$ enclosing $\lambda_{0}$ such that:

$$
m\left(\lambda_{0} ; W(\cdot)\right)=\operatorname{tr}\left(\frac{1}{2 \pi i} \int_{\Gamma} W^{\prime}(\lambda) W(\lambda)^{-1} d \lambda\right) .
$$

Remark 9. If $W(\lambda)=\lambda I-T$, where $T$ is a bounded linear operator on $\mathcal{H}$, and $\lambda_{0}$ is an isolated eigenvalue of $W(\lambda)$ in the sense of Definition 6, then $m\left(\lambda_{0} ; W(\cdot)\right)$ defined in Definition 7 is equal to the algebraic multiplicity of $\lambda_{0}$ as an eigenvalue of $T$, that is, to the dimension $\operatorname{tr} P$ of the range of the Riesz spectral projection. This follows from Theorem 8 and the identity

$$
\operatorname{tr}\left(\frac{1}{2 \pi i} \int_{\Gamma} W^{\prime}(\lambda) W(\lambda)^{-1} d \lambda\right)=\operatorname{tr}\left(\frac{1}{2 \pi i} \int_{\Gamma}(\lambda I-T)^{-1} d \lambda\right),
$$

where $\Gamma$ is a positively oriented circle centered at $\lambda_{0}$ such that $\sigma(T) \cap \Gamma=\varnothing$ and $\lambda_{0}$ is the only point in the spectrum of $T$ inside $\Gamma$.

We will now recall the setup used in [6], and several facts proved in that paper.

Hypothesis 10. (i) Suppose that $H_{0}: \operatorname{dom}\left(H_{0}\right) \rightarrow \mathcal{H}, \operatorname{dom}\left(H_{0}\right) \subseteq \mathcal{H}$ is a densely defined, closed, linear operator in $\mathcal{H}$ with nonempty resolvent set, $\rho\left(H_{0}\right) \neq \varnothing$, and that $A: \operatorname{dom}(A) \rightarrow$ $\mathcal{K}, \operatorname{dom}(A) \subseteq \mathcal{H}$ is a densely defined, closed, linear operator from $\mathcal{H}$ to $\mathcal{K}$, and $B: \operatorname{dom}(B) \rightarrow$ $\mathcal{K}, \operatorname{dom}(B) \subseteq \mathcal{H}$ is a densely defined, closed, linear operator from $\mathcal{H}$ to $\mathcal{K}$ such that

$$
\operatorname{dom}(A) \supseteq \operatorname{dom}\left(H_{0}\right), \operatorname{dom}(B) \supseteq \operatorname{dom}\left(H_{0}^{*}\right) .
$$

In the following, we denote

$$
R_{0}(z)=\left(H_{0}-z I_{\mathcal{H}}\right)^{-1}, z \in \rho\left(H_{0}\right) .
$$

(ii) For some (and hence for all) $z \in \rho\left(H_{0}\right)$, the operator $-A R_{0}(z) B^{*}$, defined on $\operatorname{dom}\left(B^{*}\right)$, has a bounded extension in $\mathcal{K}$, denoted by $K(z)$,

$$
K(z)=-\overline{A R_{0}(z) B^{*}} \in \mathcal{B}(\mathcal{K}) .
$$

(iii) $1 \in \rho\left(K\left(z_{0}\right)\right)$ for some $z_{0} \in \rho\left(H_{0}\right)$.

Lemma 11. [6, Lem. 2.2] Let $z, z_{1}, z_{2} \in \rho\left(H_{0}\right)$. Then Hypothesis 10 implies the following facts:

$$
\begin{aligned}
A R_{0}(z) \in \mathcal{B}(\mathcal{H}, \mathcal{K}), \quad \overline{R_{0}(z) B^{*}} & =\left[B\left(H_{0}^{*}-\bar{z}\right)^{-1}\right]^{*} \in \mathcal{B}(\mathcal{H}, \mathcal{K}), \\
\overline{R_{0}\left(z_{1}\right) B^{*}}-\overline{R_{0}\left(z_{2}\right) B^{*}} & =\left(z_{1}-z_{2}\right) R_{0}\left(z_{1}\right) \overline{R_{0}\left(z_{2}\right) B^{*}} \\
& =\left(z_{1}-z_{2}\right) R_{0}\left(z_{2}\right) \overline{R_{0}\left(z_{1}\right) B^{*}}
\end{aligned}
$$




$$
\begin{aligned}
K(z) & =-A \overline{\left[R_{0}(z) B^{*}\right]}, \quad K(\bar{z})^{*}=-B \overline{\left[R_{0}(\bar{z})^{*} A^{*}\right]} \\
\operatorname{ran}\left(\overline{R_{0}(z) B^{*}}\right) & \subseteq \operatorname{dom}(A), \quad \operatorname{ran}\left(\overline{R_{0}(\bar{z})^{*} A^{*}}\right) \subseteq \operatorname{dom}(B), \\
K\left(z_{1}\right)-K\left(z_{2}\right) & =\left(z_{2}-z_{1}\right) A R_{0}\left(z_{1}\right) \overline{R_{0}\left(z_{2}\right) B^{*}} \\
& =\left(z_{2}-z_{1}\right) A R_{0}\left(z_{2}\right) \overline{R_{0}\left(z_{1}\right) B^{*}}
\end{aligned}
$$

Corollary 12. The operator-valued function $K(\cdot)$ is analytic on $\rho\left(H_{0}\right)$ and

$$
K^{\prime}(z)=-A R_{0}(z)\left[B R_{0}(z)^{*}\right]^{*}, \quad z \in \rho\left(H_{0}\right) .
$$

Next, following Kato [14], one introduces

$$
R(z)=R_{0}(z)-\overline{R_{0}(z) B^{*}}\left[I_{\mathcal{K}}-K(z)\right]^{-1} A R_{0}(z), \quad z \in\left\{\zeta \in \rho\left(H_{0}\right) \mid 1 \in \rho(K(\zeta))\right\} .
$$

Theorem 13. [6, Theorem 2.3] Assume Hypothesis 10 and suppose that $z \in\left\{\zeta \in \rho\left(H_{0}\right) \mid 1 \in\right.$ $\rho(K(\zeta))\}$. Then, $R(z)$ given in (2.28) determines a densely defined, closed, linear operator $H$ in $\mathcal{H}$ by

$$
R(z)=\left(H-z I_{\mathcal{H}}\right)^{-1}
$$

Moreover,

$$
\begin{aligned}
& A R(z), B R(z)^{*} \in \mathcal{B}(\mathcal{H}, \mathcal{K}) \\
R(z) & =R_{0}(z)-\overline{R(z) B^{*}} A R_{0}(z) \\
& =R_{0}(z)-\overline{R_{0}(z) B^{*}} A R(z) .
\end{aligned}
$$

Proof. Suppose $z \in\left\{\zeta \in \rho\left(H_{0}\right) \mid 1 \in \rho(K(\zeta))\right\}$. Since, by (2.28), we have

$$
\begin{aligned}
A R(z) & =\left[I_{\mathcal{K}}-K(z)\right]^{-1} A R_{0}(z), \\
B R(z)^{*} & =\left[I_{\mathcal{K}}-K(z)^{*}\right]^{-1} B R_{0}(z)^{*},
\end{aligned}
$$

we conclude that $R(z) f=0$ implies $A R(z) f=0$, and hence $A R_{0}(z) f=0$ by (2.33). The latter implies $R_{0}(z) f=0$ by (2.28) and thus $f=0$. Consequently,

$$
\operatorname{ker}(R(z))=\{0\} .
$$

Similarly, (2.34) implies

$$
\operatorname{ker}\left(R(z)^{*}\right)=\{0\} \quad \text { and hence } \overline{\operatorname{ran} R(z)}=\mathcal{H} .
$$

Next, combining (2.28), the resolvent equation for $H_{0},(2.21),(2.22),(2.25)$ and (2.26) proves the resolvent equation

$$
R\left(z_{1}\right)-R\left(z_{2}\right)=\left(z_{1}-z_{2}\right) R\left(z_{1}\right) R\left(z_{2}\right), \quad z_{1}, z_{2} \in\left\{\zeta \in \rho\left(H_{0}\right) \mid 1 \in \rho(K(\zeta))\right\} .
$$

Thus, $R(z)$ is indeed the resolvent of a densely defined, closed, linear operator $H$ in $\mathcal{H}$ as claimed in connection with (2.29).

By (2.33) and (2.34), $A R(z) \in \mathcal{B}(\mathcal{H}, \mathcal{K})$ and $\left[B R(\bar{z})^{*}\right]^{*} \in \mathcal{B}(\mathcal{K}, \mathcal{H})$, proving (2.30). A combination of (2.28), (2.33) and (2.34) then proves (2.31) and (2.32). 
Hypothesis 14. In addition to Hypothesis 10, we impose the following assumption:

(iv) $K(z) \in \mathcal{B}_{\infty}(\mathcal{K})$ for all $z \in \rho\left(H_{0}\right)$.

Theorem 15. [6, Theorem 3.2] Assume Hypothesis 14 and let $\lambda_{0} \in \rho\left(H_{0}\right)$. Then

$$
H f=\lambda_{0} f, 0 \neq f \in \operatorname{dom}(H) \text { implies } K\left(\lambda_{0}\right) g=g
$$

where, for fixed $z_{0} \in\left\{\zeta \in \rho\left(H_{0}\right) \mid 1 \in \rho(K(\zeta))\right\}, z_{0} \neq \lambda_{0}$,

$$
\begin{aligned}
0 \neq g & =\left(I_{\mathcal{K}}-K\left(z_{0}\right)\right)^{-1} A R_{0}\left(z_{0}\right) f \\
& =\left(\lambda_{0}-z_{0}\right)^{-1} A f .
\end{aligned}
$$

Conversely,

$$
K\left(\lambda_{0}\right) g=g, 0 \neq g \in \mathcal{K} \quad \text { implies } \quad H f=\lambda_{0} f
$$

where

$$
0 \neq f=-\overline{R_{0}\left(\lambda_{0}\right) B^{*}} g \in \operatorname{dom}(H)
$$

Moreover,

$$
\operatorname{dim}\left(\operatorname{ker}\left(H-\lambda_{0} I_{\mathcal{H}}\right)\right)=\operatorname{dim}\left(\operatorname{ker}\left(I_{\mathcal{K}}-K\left(z_{0}\right)\right)\right)<\infty .
$$

In particular, let $z \in \rho\left(H_{0}\right)$, then

$$
z \in \rho(H) \text { if and only if } 1 \in \rho(K(z)) .
$$

Hypothesis 16. In addition to Hypothesis 14, we assume:

$$
\lambda_{0} \in \rho\left(H_{0}\right) \cap\{\lambda \in \mathbb{C} \mid \lambda \text { is an isolated eigenvalue of } H\} .
$$

Hypothesis 16 implies that $I_{\mathcal{K}}-K(\cdot)$ is an operator-valued function analytic in some neighborhood of $\lambda_{0}$. Moreover, $\lambda_{0}$ is an eigenvalue of finite type of $I_{\mathcal{K}}-K(\cdot)$ as described in Definition 6. Therefore, the algebraic multiplicity $m\left(\lambda_{0} ; I_{\mathcal{K}}-K(\cdot)\right)$ from Definition 7 is well-defined.

Lemma 17. [9, Section XI.9] Let $G_{1}$ and $G_{2}$ be $\mathcal{B}(\mathcal{H})$-valued operator functions which are finitely meromorphic at $\lambda_{0}$. Then $G_{1} G_{2}$ and $G_{2} G_{1}$ are finitely meromorphic at $\lambda_{0}$ and

$$
\operatorname{tr} \Xi\left(G_{1} G_{2}\right)(\lambda)=\operatorname{tr} \Xi\left(G_{2} G_{1}\right)(\lambda), \lambda \neq \lambda_{0},
$$

where $\Xi(G)(\lambda)$ stands for the principal part of $G$ at $\lambda_{0}$.

We will now apply the Gohberg-Rouche Theorem 8 in the setup of [6] to show equality of the algebraic multiplicity of the eigenvalue $\lambda_{0}$ of finite type of $I_{\mathcal{K}}-K(\cdot)$, see Definition 7, and the "usual" algebraic multiplicity $m\left(\lambda_{0} ; H\right)$ of the eigenvalue $\lambda_{0}$ of $H$. We recall that $m\left(\lambda_{0} ; H\right)$ is defined as the dimension of the range of the Riesz spectral projection:

$$
m\left(\lambda_{0} ; H\right)=-\frac{1}{2 \pi i} \operatorname{tr}\left(\int_{\Gamma} d \lambda R(\lambda)\right), \quad \text { where } \quad R(\lambda)=(H-\lambda)^{-1}
$$


Theorem 18. Assume Hypothesis 16. Then

$$
m\left(\lambda_{0} ; I_{\mathcal{K}}-K(\cdot)\right)=m\left(\lambda_{0} ; H\right) .
$$

Proof. First of all, using formula (2.16), (2.27), (2.34) and (2.46), we infer:

$$
\begin{aligned}
m\left(\lambda_{0} ; I_{\mathcal{K}}-K(\cdot)\right) & =\frac{1}{2 \pi i} \operatorname{tr}\left(\int_{\Gamma} d \lambda\left(I_{\mathcal{K}}-K(\lambda)\right)^{\prime}\left(I_{\mathcal{K}}-K(\lambda)\right)^{-1}\right) \\
& =\frac{1}{2 \pi i} \operatorname{tr}\left(\int_{\Gamma} d \lambda \Xi\left\{\left(I_{\mathcal{K}}-K(\lambda)\right)^{\prime}\left(I_{\mathcal{K}}-K(\lambda)\right)^{-1}\right\}\right) \\
& =\frac{1}{2 \pi i} \operatorname{tr}\left(\int_{\Gamma} d \lambda \Xi\left\{A R_{0}(z)\left[B R_{0}(z)^{*}\right]^{*}\left(I_{\mathcal{K}}-K(\lambda)\right)^{-1}\right\}\right) \\
& =\frac{1}{2 \pi i} \operatorname{tr}\left(\int_{\Gamma} d \lambda \Xi\left\{A R_{0}(z)\left[B R(z)^{*}\right]^{*}\right\}\right) \\
& =\frac{1}{2 \pi i}\left(\int_{\Gamma} d \lambda \operatorname{tr} \Xi\left\{A R_{0}(z)\left[B R(z)^{*}\right]^{*}\right\}\right) \\
& =\frac{1}{2 \pi i}\left(\int_{\Gamma} d \lambda \operatorname{tr} \Xi\left\{\left[B R(z)^{*}\right]^{*} A R_{0}(z)\right\}\right) .
\end{aligned}
$$

On the other hand, using (2.28) and (2.34), we infer:

$$
\begin{aligned}
m\left(\lambda_{0} ; H\right) & =-\frac{1}{2 \pi i} \operatorname{tr}\left(\int_{\Gamma} d \lambda\left[R(\lambda)-R_{0}(\lambda)\right]\right) \\
& =-\frac{1}{2 \pi i} \operatorname{tr}\left(\int_{\Gamma} d \lambda \Xi\left\{R(\lambda)-R_{0}(\lambda)\right\}\right) \\
& =\frac{1}{2 \pi i} \operatorname{tr}\left(\int_{\Gamma} d \lambda \Xi\left\{\left[B R_{0}(\lambda)^{*}\right]^{*}\left(I_{\mathcal{K}}-K(\lambda)\right)^{-1} A R_{0}(\lambda)\right\}\right) \\
& =\frac{1}{2 \pi i} \operatorname{tr}\left(\int_{\Gamma} d \lambda \Xi\left\{\left[B R(z)^{*}\right]^{*} A R_{0}(z)\right\}\right) \\
& =\frac{1}{2 \pi i}\left(\int_{\Gamma} d \lambda \operatorname{tr} \Xi\left\{\left[B R(z)^{*}\right]^{*} A R_{0}(z)\right\}\right) .
\end{aligned}
$$

Combining (2.49) and (2.50), we obtain the desired identity.

Remark 19. Assume Hypothesis 16. Then, it follows from Theorem 15 that $\lambda_{0}$ is an isolated eigenvalue of $H$ of finite geometric multiplicity. Moreover, from Theorem 18 it follows that $\lambda_{0}$ is also an isolated eigenvalue of $H$ of finite algebraic multiplicity. 
Hypothesis 20. In addition to Hypothesis 16, we assume the following condition:

(v) For some $p \in \mathbb{N}$, we have $K(z) \in \mathcal{B}_{p}(\mathcal{K})$ for all $z \in \rho\left(H_{0}\right)$.

Theorem 21. Assume Hypothesis 20. Then, the following holds:

$$
\begin{aligned}
\operatorname{det}_{p}\left(I_{\mathcal{K}}-K(\lambda)\right) & =\left(\lambda-\lambda_{0}\right)^{m\left(\lambda_{0} ; I_{\mathcal{K}}-K(\cdot)\right)} S(\lambda) \\
& =\left(\lambda-\lambda_{0}\right)^{m\left(\lambda_{0} ; H\right)} S(\lambda), \quad S\left(\lambda_{0}\right) \neq 0 .
\end{aligned}
$$

Proof. The first equality follows directly from Theorem 4 and Corollary 5 applied to $W(\lambda)=$ $I_{\mathcal{K}}-K(\lambda)$. Hence, from (2.9),

$$
\begin{aligned}
\operatorname{det}_{p}\left(I_{\mathcal{K}}-K(\lambda)\right) & =\operatorname{det}_{p}(D(\lambda)) S_{1}(\lambda) \\
& =\left(\lambda-\lambda_{0}\right)^{m\left(\lambda_{0} ; I_{\mathcal{K}}-K(\cdot)\right)} S(\lambda), \quad S\left(\lambda_{0}\right) \neq 0 .
\end{aligned}
$$

Equality (2.52) follows from Theorem 18.

\section{Applications}

\subsection{The Schrödinger Equation}

Let us consider the Schrödinger equation

$$
-\phi^{\prime \prime}(x)+V(x) \phi(x)=\lambda \phi(x)
$$

with the potential $V \in L^{1}(\mathbb{R} ; d x)$. We introduce the closed operators in $L^{2}(\mathbb{R} ; d x)$ defined by

$$
\begin{aligned}
& H_{0} f=-f^{\prime \prime}, \quad f \in \operatorname{dom}\left(H_{0}\right), \quad\left(\operatorname{dom}\left(H_{0}\right),\|\cdot\|_{H_{0}}\right)=W_{2}^{2}(\mathbb{R}), \\
& H f=-f^{\prime \prime}+V f, \\
& \quad f \in \operatorname{dom}(H)=\left\{g \in L^{2}(\mathbb{R} ; d x) \mid g, g^{\prime} \in A C_{\text {loc }}(\mathbb{R}) ;\left(-g^{\prime \prime}+V g\right) \in L^{2}(\mathbb{R} ; d x)\right\} .
\end{aligned}
$$

Also, we introduce the factorization

$$
V=u(x) v(x), u(x)=\operatorname{sign}(V(x))|V(x)|^{1 / 2}, v(x)=|V(x)|^{1 / 2}, x \in \mathbb{R} .
$$

Finally, we introduce the integral operator $K(\lambda)$ in $L^{2}(\mathbb{R} ; d x)$ with the integral kernel

$$
K\left(\lambda, x, x^{\prime}\right)=-v(x) M^{\vee}\left(\lambda, x-x^{\prime}\right) u\left(x^{\prime}\right), \quad \lambda \in \mathbb{C} \backslash \sigma\left(H_{0}\right),
$$

where $M(\lambda, \eta)=\left(\eta^{2}-\lambda\right)^{-1}$, and ${ }^{\vee}$ denotes the inverse Fourier transform with respect to the variable $\eta \in \mathbb{R}$. The following result is, of course, well-known; we recall its proof to emphasize its similarity with the proof of Theorems 33 and 41 below. 
Theorem 22. [19, Theorem 4.1.] Suppose $V \in L^{1}(\mathbb{R} ; d x)$ and let $\lambda \in \mathbb{C} \backslash \mathbb{R}_{+}$. Then $K(\lambda) \in$ $\mathcal{B}_{2}\left(L^{2}(\mathbb{R} ; d x)\right)$.

Proof. Using [16, Theorem VI.23], the assertion in the theorem follows from the formula for the $\mathcal{B}_{2}$-norm of an integral operator:

$$
\begin{aligned}
& \|K(\lambda)\|_{\mathcal{B}_{2}\left(L^{2}(\mathbb{R} ; d x)\right.}=\iint_{\mathbb{R} \times \mathbb{R}} d x d x^{\prime}\left|K\left(\lambda, x, x^{\prime}\right)\right|^{2}= \\
= & \iint_{\mathbb{R} \times \mathbb{R}} d x d x^{\prime}\left|-v(x) M^{\vee}\left(\lambda, x-x^{\prime}\right) u\left(x^{\prime}\right)\right|^{2}<\infty .
\end{aligned}
$$

The last inequality follows from $u, v \in L^{2}(\mathbb{R})$ because $M^{\vee}(\lambda, \cdot) \in L^{\infty}(\mathbb{R} ; d x)$ by $M(\lambda, \cdot) \in$ $L^{1}(\mathbb{R} ; d x)$ and the Riemann-Lebesgue lemma.

Remark 23. Assume $V \in L^{1}(\mathbb{R} ; d x)$ and let $\lambda \in \mathbb{C} \backslash \sigma\left(H_{0}\right)$. Since $M(\lambda, \cdot) \in L^{2}(\mathbb{R} ; d \eta) \cap$ $L^{\infty}(\mathbb{R} ; d \eta)$, by [17, Theorem IX.29] the operator $K(\lambda)$ coincides with $-M_{v}\left(H_{0}-\lambda\right)^{-1} M_{u}$ on the domain of $M_{u}$, where $M_{v}, M_{u}$ are the operators of multiplication by $v$, $u$ with maximal domains. Hence, $K(\lambda)=-\overline{M_{v}\left(H_{0}-\lambda\right)^{-1} M_{u}} \in \mathcal{B}_{2}\left(L^{2}(\mathbb{R} ; d x)\right)$. From now on, we will use the notation $K(\lambda)$ also for the operator $-\overline{M_{v}\left(H_{0}-\lambda\right)^{-1} M_{u}}$.

The Schrödinger equation (3.1) is equivalent to the first order system

$$
\Psi^{\prime}(x)=\left[\begin{array}{cc}
0 & 1 \\
V(x)-\lambda & 0
\end{array}\right] \Psi(x), \Psi=\left[\begin{array}{c}
\phi^{\prime} \\
\phi
\end{array}\right] .
$$

Introduce the correspoding first order operator $T(\lambda)$ and matrices $A$ and $B$ :

$$
\begin{aligned}
& T(\lambda)=\partial_{x}+\left[\begin{array}{cc}
0 & -1 \\
\lambda & 0
\end{array}\right],\left(\operatorname{dom}(T(\lambda)),\|\cdot\|_{T(\lambda)}\right)=W_{2}^{1}(\mathbb{R}) \oplus W_{2}^{1}(\mathbb{R}), \lambda \in \mathbb{C} \backslash \mathbb{R}_{+}, \\
& A(\lambda)=\left[\begin{array}{cc}
0 & 1 \\
-\lambda & 0
\end{array}\right], B(x)=\left[\begin{array}{cc}
0 & 0 \\
V(x) & 0
\end{array}\right] .
\end{aligned}
$$

Then, the first order system (3.5) can be rewritten as follows:

$$
\Psi^{\prime}(x)=(A(\lambda)+B(x)) \Psi(x) .
$$

Lemma 24. The operator $T(\lambda)$, with $\lambda \in \mathbb{C} \backslash \mathbb{R}_{+}$, has a bounded inverse given by

$$
T(\lambda)^{-1}=\left[\begin{array}{cc}
-\partial_{x}\left(-\partial_{x x}^{2}-\lambda\right)^{-1} & -\left(-\partial_{x x}^{2}-\lambda\right)^{-1} \\
\lambda\left(-\partial_{x x}^{2}-\lambda\right)^{-1} & -\partial_{x}\left(-\partial_{x x}^{2}-\lambda\right)^{-1}
\end{array}\right] .
$$

Proof. Clearly,

$$
\left(i \eta I_{2 \times 2}+\left[\begin{array}{cc}
0 & -1 \\
\lambda & 0
\end{array}\right]\right)^{-1}=-\frac{1}{\eta^{2}-\lambda}\left[\begin{array}{cc}
i \eta & 1 \\
-\lambda & i \eta
\end{array}\right], \eta \in \mathbb{R}
$$


Taking the Fourier transform in $\eta$-variable proves the assertion.

Introduce the matrices $\widetilde{u}(x), \widetilde{v}(x)$ and the operator $\mathbb{K}(\lambda)$ as follows:

$$
\begin{aligned}
\widetilde{u}(x) & =\left[\begin{array}{cc}
0 & 0 \\
u(x) & 0
\end{array}\right], \widetilde{v}(x)=\left[\begin{array}{cc}
v(x) & 0 \\
0 & 0
\end{array}\right], \\
\mathbb{K}(\lambda) & =-\overline{M_{\widetilde{v}} T(\lambda)^{-1} M_{\widetilde{u}}}, \quad \lambda \in \mathbb{C} \backslash \mathbb{R}_{+} .
\end{aligned}
$$

Here, the operator $M_{\widetilde{v}} T(\lambda)^{-1} M_{\widetilde{u}}$ is originaly defined on the (maximal) domain of the operator $M_{\widetilde{u}}$ of multiplication by $\widetilde{u}$. We will see in (3.12) that $\mathbb{K}(\lambda) \in \mathcal{B}\left(L^{2}(\mathbb{R} ; d x)^{2}\right)$. Also, $B(x)$ in (3.6) has the following representation: $B(x)=\widetilde{u}(x) \widetilde{v}(x)$.

Lemma 25. Suppose that $V \in L^{1}(\mathbb{R} ; d x)$ and $\lambda \in \mathbb{C} \backslash \mathbb{R}_{+}$. Then $\mathbb{K}(\lambda) \in \mathcal{B}_{2}\left(L^{2}(\mathbb{R} ; d x)^{2}\right)$ and the following equality holds:

$$
\operatorname{det}_{2}\left(I_{L^{2}(\mathbb{R} ; d x)^{2}}-\mathbb{K}(\lambda)\right)=\operatorname{det}_{2}\left(I_{L^{2}(\mathbb{R} ; d x)}+K(\lambda)\right) .
$$

Proof. Using (3.8), we arrive at the following identity:

$$
\mathbb{K}(\lambda)=\overline{\left[\begin{array}{cc}
-v & 0 \\
0 & 0
\end{array}\right] T(\lambda)^{-1}\left[\begin{array}{ll}
0 & 0 \\
u & 0
\end{array}\right]}=\overline{\left[\begin{array}{cc}
v(x)\left(-\partial_{x x}^{2}-\lambda\right)^{-1} u(x) & 0 \\
0 & 0
\end{array}\right]}=\left[\begin{array}{cc}
-K(\lambda) & 0 \\
0 & 0
\end{array}\right] .
$$

The required assertion now follows from Theorem 22 and (3.12).

We now recall that the eigenvalues of $H$ are zeros of the Evans function $\mathbb{E}$ associated with the first order system (3.7), see, e.g., $[1,4,15,18,20]$. Our next goal is to describe the relations of the algebraic multiplicity $m\left(\lambda_{0} ; H\right)$ of the eigenvalue $\lambda_{0}$ of the operator $H$ defined in (3.2), the algebraic multiplicity $m\left(\lambda_{0} ; I-K(\cdot)\right)$ of the operator-valued function $K(\cdot)$ at $\lambda_{0}$ (as defined in Definition 7), and the multiplicity $m\left(\lambda_{0} ; \mathbb{E}(\cdot)\right)$ of the zero at $\lambda_{0}$ of the Evans function $\mathbb{E}$.

Let us first recall the definition of the Evans function, see, e.g., [18]. Consider a first order system

$$
\frac{d}{d \xi} u=\mathbb{M}(\xi, \lambda) u, u \in \mathbb{C}^{n}, \xi \in \mathbb{R},
$$

where $\mathbb{M}$ is a sum of an $L^{\infty}\left(\mathbb{R} ; \mathbb{C}^{n \times n}\right)$ - and an $L^{1}\left(\mathbb{R} ; \mathbb{C}^{n \times n}\right)$-function of $\xi$ analytic in $\lambda$ in some domain. We say that $\lambda$ is not in the essential spectrum $\sigma_{\text {ess }}$ of (3.13) if the operator $\partial_{\xi}-\mathbb{M}(\cdot, \lambda)$ is Fredholm with zero index. If this is the case then equation (3.13) has exponential dichotomies on $\mathbb{R}^{+}$and $\mathbb{R}^{-}$with projections $P_{+}(\xi ; \lambda)$ and $P_{-}(\xi ; \lambda)$, respectively (this means that $\operatorname{ran}\left(P_{+}(0 ; \lambda)\right.$ ), respectively, $\operatorname{ker}\left(P_{+}(0 ; \lambda)\right)$ is the set of the initial data of the solutions of $(3.13)$ on $\mathbb{R}^{+}$that decay, respectively, grow exponentially at $+\infty$, while $\operatorname{ker}\left(P_{-}(0 ; \lambda)\right)$, respectively, $\operatorname{ran}\left(P_{-}(0 ; \lambda)\right)$ is the set of the initial data of the solutions of (3.13) on $\mathbb{R}^{-}$that decay, respectively, grow exponentially at $-\infty)$. Moreover, the Morse indices of the dichotomies are equal, that is, $\operatorname{dim} \operatorname{ker}\left(P_{+}(0 ; \lambda)\right)=$ $\operatorname{dim} \operatorname{ker}\left(P_{-}(0 ; \lambda)\right)$, see Palmer's theorem in [18]. Let $\Omega$ be a simply-connected subset of $\mathbb{C} \backslash \sigma_{\text {ess }}$. Then the Morse index $\operatorname{dim} \operatorname{ker}\left(P_{+}(0 ; \lambda)\right)=\operatorname{dim} \operatorname{ker}\left(P_{-}(0 ; \lambda)\right)$ is constant for $\lambda \in \Omega$; let us denote it by $k$. We choose ordered bases $\left[u_{1}(\lambda), \ldots, u_{k}(\lambda)\right]$ and $\left[u_{k+1}(\lambda), \ldots, u_{n}(\lambda)\right]$ of the subspaces 
$\operatorname{ker}\left(P_{-}(0 ; \lambda)\right)$ and $\operatorname{ran}\left(P_{+}(0 ; \lambda)\right)$, respectively. We can choose the basis vectors such that they are analytic in $\lambda$.

Definition 26. The Evans function $\mathbb{E}$ is defined by

$$
\mathbb{E}(\lambda)=\operatorname{det}\left[u_{1}(\lambda), \ldots, u_{n}(\lambda)\right]
$$

We note that the Evans function depends on the choice of the basis vectors $u_{j}(\lambda)$. As shown in [5], if the first order system (3.13) has an additional perturbation structure as in (3.7), then the Evans function can be chosen to agree with the (modified) Fredholm determinant of the operator $I-\mathbb{K}(\lambda)$. Specifically, the basis vectors $u_{j}(\lambda)$ can be chosen as the columns of the generalized matrix-valued Jost solutions of the first order system (3.7). The definition of the generalized matrix-valued Jost solution can be found in [5]. The paper [5] contains the following formula:

$$
\operatorname{det}_{2}\left(I_{L^{2}(\mathbb{R} ; d x)^{2}}-\mathbb{K}(\lambda)\right)=e^{\Theta(\lambda)} \mathbb{E}(\lambda)
$$

where $\Theta(\lambda)$ is some number explicitly computed in [5].

In what follows we always assume that the Evans function is selected such that (3.15) holds. If $\mathbb{E}\left(\lambda_{0}\right)=0$ then we denote by $m\left(\lambda_{0} ; \mathbb{E}(\cdot)\right)$ the multiplicity of $\lambda_{0}$ as a zero of the function $\mathbb{E}$ such that $\mathbb{E}(\lambda)=\left(\lambda-\lambda_{0}\right)^{m\left(\lambda_{0} ; \mathbb{E}(\cdot)\right)} S(\lambda), S\left(\lambda_{0}\right) \neq 0$.

Hypothesis 27. We assume that $V \in L^{1}(\mathbb{R})$ and

$$
\lambda_{0} \in\left\{\mathbb{C} \backslash \mathbb{R}_{+}\right\} \cap\{\lambda \in \mathbb{C} \mid \lambda \text { is an isolated eigenvalue of } H\} .
$$

Theorem 28. Assume Hypothesis 27 and let $\mathbb{E}(\cdot)$ be the Evans function for the perturbed equation (3.7). Then

$$
m\left(\lambda_{0} ; \mathbb{E}(\cdot)\right)=m\left(\lambda_{0} ; I_{L^{2}(\mathbb{R} ; d x)}-K(\cdot)\right)=m\left(\lambda_{0} ; H\right) .
$$

Proof. This follows from formula (3.15), Lemma 25, Theorem 21 and Theorem 18.

\subsection{Degenerate Reaction-Diffusion Systems}

Let $D$ be a diagonal $n \times n$ matrix with the diagonal entries $d_{1}, \ldots d_{\ell}>0$ and $d_{\ell+1}=\ldots=d_{n}=0$, and $F: \mathbb{R}^{n} \rightarrow \mathbb{R}^{n}$ be a smooth function. We consider the system of reaction diffusion equations

$$
U_{t}=D U_{x x}+F(U), \quad x \in \mathbb{R}, U \in \mathbb{R}^{n} .
$$

In the moving coordinate frame $\xi=x-c t$, with some $c>0$, system (3.18) is given by

$$
U_{t}=D U_{\xi \xi}+c U_{\xi}+F(U), \quad \xi \in \mathbb{R}, U \in \mathbb{R}^{n} .
$$

Suppose that $Q=Q(\xi)$ is a traveling wave for (3.18), that is, is a stationary solution of (3.19):

$$
D Q_{\xi \xi}(\xi)+c Q_{\xi}(\xi)+F(Q(\xi))=0, \quad \xi \in \mathbb{R} .
$$


The eigenvalue problem associated with the linearization of (3.19) about $Q$ is given by

$$
D U_{\xi \xi}+c U_{\xi}+F^{\prime}(Q) U=\lambda U
$$

here and below $F^{\prime}=\partial F$ denotes the differential of $F$.

We decompose $U(\xi), F^{\prime}(Q(\xi))$ in the following way:

$$
\begin{aligned}
U & =\left[U_{1}(\xi), U_{2}(\xi)\right]^{\top} \in \mathbb{R}^{\ell} \oplus \mathbb{R}^{n-\ell} \\
F^{\prime}(Q(\xi)) & =\left[\begin{array}{ll}
F_{11}^{\prime}(Q(\xi)) & F_{12}^{\prime}(Q(\xi)) \\
F_{21}^{\prime}(Q(\xi)) & F_{22}^{\prime}(Q(\xi))
\end{array}\right]: \mathbb{R}^{\ell} \oplus \mathbb{R}^{n-\ell} \rightarrow \mathbb{R}^{\ell} \oplus \mathbb{R}^{n-\ell} .
\end{aligned}
$$

Let $D_{\ell}$ be the diagonal $\ell \times \ell$ matrix with the diagonal entries $d_{1}, \ldots d_{\ell}$, and denote $W_{1}=\frac{d}{d \xi} U_{1}$. Then the eigenvalue problem (3.21) can be recast as follows:

$$
\left[\begin{array}{ccc}
\partial_{\xi} & 0_{\ell \times(n-\ell)} & -I_{\ell \times \ell} \\
c^{-1} F_{21}^{\prime}(Q(\xi)) & c^{-1}\left(-\lambda+F_{22}^{\prime}(Q(\xi))\right)+\partial_{\xi} & 0_{(n-\ell) \times \ell} \\
D_{\ell}^{-1}\left(-\lambda+F_{11}^{\prime}(Q(\xi))\right) & D_{\ell}^{-1} F_{12}^{\prime}(Q(\xi)) & c D_{\ell}^{-1}+\partial_{\xi}
\end{array}\right]\left[\begin{array}{c}
U_{1} \\
U_{2} \\
W_{1}
\end{array}\right]=0 .
$$

Hypothesis 29. Suppose that the traveling wave $Q$ is a pulse, that is, the following limit exists:

$$
\lim _{|\xi| \rightarrow \infty} Q(\xi)=Q(\infty) \in \mathbb{R}^{n}
$$

Assume Hypothesis 29. Denoting by $W_{2}^{k}$ the Sobolev space of $k$ times differentiable functions with $L^{2}$-derivatives, we introduce the operators $T(\lambda), H_{0}, H$, and the matrices $A, B, V$ as follows:

$$
T(\lambda)=\left[\begin{array}{ccc}
\partial_{\xi} & 0_{\ell \times(n-\ell)} & -I_{\ell \times \ell} \\
c^{-1} F_{21}^{\prime}(Q(\infty)) & c^{-1}\left(-\lambda+F_{22}^{\prime}(Q(\infty))\right)+\partial_{\xi} & 0_{(n-\ell) \times \ell} \\
D_{\ell}^{-1}\left(-\lambda+F_{11}^{\prime}(Q(\infty))\right) & D_{\ell}^{-1} F_{12}^{\prime}(Q(\infty)) & c D_{\ell}^{-1}+\partial_{\xi}
\end{array}\right]
$$

with the domain $\left(\operatorname{dom}(T(\lambda)),\|\cdot\|_{T(\lambda)}\right)=W_{2}^{1}(\mathbb{R})^{n+\ell}$,

$$
H_{0}=\left[\begin{array}{cc}
D_{\ell} \partial_{\xi \xi}^{2}+c \partial_{\xi} & 0_{\ell \times(n-\ell)} \\
0_{(n-\ell) \times \ell} & c \partial_{\xi}
\end{array}\right]+F^{\prime}(Q(\infty)),
$$

with the domain $\left(\operatorname{dom}\left(H_{0}\right),\|\cdot\|_{H_{0}}\right)=W_{2}^{2}(\mathbb{R})^{\ell} \oplus W_{2}^{1}(\mathbb{R})^{n-\ell}$,

$$
\begin{gathered}
H=H_{0}+V \text {, with } V(\xi)=F^{\prime}(Q(\xi))-F^{\prime}(Q(\infty)) \text { and the domain } \\
\operatorname{dom}(H)=\left\{f=(g, h)^{\top} \in L^{2}(\mathbb{R} ; d \xi)^{\ell} \oplus L^{2}(\mathbb{R} ; d \xi)^{n-\ell} \mid\right. \\
\left.\qquad g, g^{\prime} \in A C_{\mathrm{loc}}(\mathbb{R})^{\ell}, h \in A C_{\mathrm{loc}}(\mathbb{R})^{n-\ell} ; H_{0} f+V f \in L^{2}(\mathbb{R} ; d \xi)^{n}\right\},
\end{gathered}
$$




$$
\begin{gathered}
A(\lambda)=\left[\begin{array}{ccc}
0 & 0 & I \\
-c^{-1} F_{21}^{\prime}(Q(\infty)) & c^{-1}\left(\lambda-F_{22}^{\prime}(Q(\infty))\right) & 0 \\
D_{\ell}^{-1}\left(\lambda-F_{11}^{\prime}(Q(\infty))\right) & -D_{\ell}^{-1} F_{12}^{\prime}(Q(\infty)) & -c D_{\ell}^{-1}
\end{array}\right], \\
B(\xi)=\left[\begin{array}{ccc}
0 & 0 \\
c^{-1}\left(-F_{21}^{\prime}(Q(\xi))+F_{21}^{\prime}(Q(\infty))\right) & c^{-1}\left(-F_{22}^{\prime}(Q(\xi))+F_{22}^{\prime}(Q(\infty))\right) & 0 \\
D_{\ell}^{-1}\left(-F_{11}^{\prime}(Q(\xi))+F_{11}^{\prime}(Q(\infty))\right) & D_{\ell}^{-1}\left(-F_{12}^{\prime}(Q(\xi))+F_{12}^{\prime}(Q(\infty))\right) & 0
\end{array}\right] .
\end{gathered}
$$

Then, the eigenvalue problem (3.22) can be recast as

$$
\Psi^{\prime}(\xi)=(A(\lambda)+B(\xi)) \Psi(\xi)
$$

where $\Psi(\xi)=\left[U_{1}(\xi), U_{2}(\xi), W_{1}(\xi)\right]^{\top}$. Passing to the Fourier transform yields the following fact.

Lemma 30. Let $H_{0}$ be as in (3.25). Then

$$
\rho\left(H_{0}\right)=\left\{\lambda \in \mathbb{C}\left|\inf _{\eta \in \mathbb{R}}\right| \operatorname{det} N(\lambda, \eta) \mid \neq 0\right\},
$$

where we denote:

$$
N(\lambda, \eta)=\left[\begin{array}{cc}
-\eta^{2} D_{\ell}+i c \eta+F_{11}^{\prime}(Q(\infty))-\lambda & F_{12}^{\prime}(Q(\infty)) \\
F_{21}^{\prime}(Q(\infty)) & i c \eta+F_{22}^{\prime}(Q(\infty))-\lambda
\end{array}\right]
$$

For $\lambda \in \rho\left(H_{0}\right)$ one can express $\left(H_{0}-\lambda\right)^{-1}$ in terms of the multiplication operator:

$$
\left(H_{0}-\lambda\right)^{-1}=\mathcal{F}^{-1} M(\lambda, \cdot) \mathcal{F}
$$

where $\mathcal{F}$ is the Fourier transform, and we denote

$$
M(\lambda, \eta)=\left[\begin{array}{cc}
-\eta^{2}+i c \eta+F_{11}^{\prime}(Q(\infty))-\lambda & F_{12}^{\prime}(Q(\infty)) \\
F_{21}^{\prime}(Q(\infty)) & i c \eta+F_{22}^{\prime}(Q(\infty))-\lambda
\end{array}\right]^{-1}
$$

Remark 31. Since $\lambda \in \rho\left(H_{0}\right)$, by Lemma 30 the determinant of $N(\lambda, \eta)$ is separated from zero uniformly in $\eta \in \mathbb{R}$. Moreover, each element $m_{i j}$ of the matrix $M(\lambda, \eta)$ is of the form $p_{i j}(\lambda, \eta) / \operatorname{det}(N(\lambda, \eta))$, where $p_{i j}(\lambda, \eta)$ and $\operatorname{det}(N(\lambda, \eta))$ are polynomials in $\eta$, and

$$
\operatorname{deg}\left(p_{i j}(\lambda, \eta)\right)+1 \leq \operatorname{deg}(\operatorname{det}(N(\lambda, \eta)))
$$

Hence, $M(\lambda, \cdot) \in L^{2}(\mathbb{R} ; d \eta)^{n \times n} \cap L^{\infty}(\mathbb{R} ; d \eta)^{n \times n}$. So, we conclude that the Fourier transform of $M(\lambda, \cdot)$ is well-defined in $L^{2}$-sense.

Hypothesis 32. In addition to Hypothesis 29, we assume that $F^{\prime}(Q(\cdot))-F^{\prime}(Q(\infty)) \in L^{1}(\mathbb{R})^{n \times n}$. 
Let $\widetilde{U}(\xi)$ and $\left|F^{\prime}(Q(\xi))-F^{\prime}(Q(\infty))\right|$ denote the $n \times n$ matrices in the polar decomposition of $F^{\prime}(Q(\xi))-F^{\prime}(Q(\infty))$. We introduce the matrices

$$
u(\xi)=\widetilde{U}(\xi)\left|F^{\prime}(Q(\xi))-F^{\prime}(Q(\infty))\right|^{1 / 2}, v(\xi)=\left|F^{\prime}(Q(\xi))-F^{\prime}(Q(\infty))\right|^{1 / 2} .
$$

Assume Hypothesis 32. Then $u, v \in L^{2}(\mathbb{R})^{n \times n}$. Next, using Remark 31, we introduce the integral operator $K(\lambda)$ in $L^{2}(\mathbb{R} ; d \xi)^{n}$ with the integral kernel

$$
K\left(\lambda, \xi, \xi^{\prime}\right)=-v(\xi) M^{\vee}\left(\lambda, \xi-\xi^{\prime}\right) u\left(\xi^{\prime}\right) .
$$

Theorem 33. Assume Hypothesis 32 and let $\lambda \in \mathbb{C} \backslash \sigma\left(H_{0}\right)$. Then $K(\lambda) \in \mathcal{B}_{2}\left(L^{2}(\mathbb{R} ; d \xi)^{n}\right)$.

Proof. First, we claim that $M^{\vee}(\lambda, \cdot) \in L^{\infty}(\mathbb{R} ; d \xi)^{n \times n}$. Indeed, by Remark 31 each element $m_{i j}$ of the matrix $M(\lambda, \eta)$ is of the form $p_{i j}(\lambda, \eta) / \operatorname{det}(N(\lambda, \eta))$, where $p_{i j}(\lambda, \eta)$ and $\operatorname{det}(N(\lambda, \eta))$ are polynomials in $\eta$. Denoting by $\eta_{k}$ the roots of $N(\lambda, \cdot)$, we can decompose $m_{i j}$ as the follows:

$$
m_{i j}(\lambda, \eta)=\sum_{k=1}^{d} \sum_{l=1}^{s_{k}} \frac{a_{i j k l}}{\left(\eta-\eta_{k}\right)^{l}}, a_{i j k l} \in \mathbb{C}, \operatorname{Im}\left(\eta_{k}\right) \neq 0,
$$

where $d$ is the number of different roots of $\operatorname{det}(N(\lambda, \cdot))$ and $s_{k}$ is the multiplicity of the root $\eta_{k}$ of $\operatorname{det}(N(\lambda, \cdot))$. Note that some of $a_{i j k l}$ might be zero. If $l>1$ then

$$
a_{i j k l} /\left(\cdot-\eta_{k}\right)^{l} \in L^{1}(\mathbb{R} ; d \eta) \text { and }\left(a_{i j k l} /\left(\eta-\eta_{k}\right)^{l}\right)^{\vee} \in L^{\infty}(\mathbb{R} ; d \xi)
$$

by the Riemann-Lebesgue lemma. If $l=1$ then $\left(a_{i j k l} /\left(\eta-\eta_{k}\right)^{l}\right)^{\vee} \in L^{\infty}(\mathbb{R} ; d \xi)$ since

$$
\begin{aligned}
& \text { if } \operatorname{Re}(\lambda)<0 \text { then }\left(\frac{1}{i \eta-\lambda}\right)^{\vee}(\xi)= \begin{cases}\sqrt{2 \pi} \mathrm{e}^{\lambda \xi}, & \xi \geq 0 ; \\
0, & \xi<0 ;\end{cases} \\
& \text { if } \operatorname{Re}(\lambda)>0 \text { then }\left(\frac{1}{i \eta-\lambda}\right)^{\vee}(\xi)= \begin{cases}0, & \xi \geq 0 ; \\
\sqrt{2 \pi} \mathrm{e}^{\lambda \xi}, & \xi<0 .\end{cases}
\end{aligned}
$$

Combining the cases $l>1$ and $l=1$, we justify the claim $M^{\vee}(\lambda, \cdot) \in L^{\infty}(\mathbb{R} ; d \xi)^{n \times n}$. The assertion in the theorem now follows from the well-known formula for the $\mathcal{B}_{2}$-norm of an integral operator, see [2, Theorem 11.3.6]:

$$
\begin{aligned}
& \|K(\lambda)\|_{\mathcal{B}_{2}\left(L^{2}(\mathbb{R} ; d \xi)^{n}\right)}^{2}=\iint_{\mathbb{R} \times \mathbb{R}} d \xi d \xi^{\prime}\left\|K\left(\lambda, \xi, \xi^{\prime}\right)\right\|_{\mathbb{C}^{n \times n}}^{2}= \\
& =\iint_{\mathbb{R} \times \mathbb{R}} d \xi d \xi^{\prime}\left\|-v(\xi) M^{\vee}\left(\lambda, \xi-\xi^{\prime}\right) u\left(\xi^{\prime}\right)\right\|_{\mathbb{C}^{n \times n}}^{2}<\infty .
\end{aligned}
$$

The last inequality holds since $u, v \in L^{2}(\mathbb{R})^{n \times n}$ and $M^{\vee}(\lambda, \cdot) \in L^{\infty}(\mathbb{R} ; d \xi)^{n \times n}$. 
Remark 34. Assume Hypothesis 32 and let $\lambda \in \mathbb{C} \backslash \sigma\left(H_{0}\right)$. Since $M(\lambda, \cdot) \in L^{2}(\mathbb{R} ; d \eta)^{n \times n} \cap$ $L^{\infty}(\mathbb{R} ; d \eta)^{n \times n}$, it follows by [17, Theorem IX.29] that $f \in \operatorname{dom}\left(M_{u}\right)$ yields $K(\lambda) f=-M_{v}\left(H_{0}-\right.$ $\lambda)^{-1} M_{u} f$; here $M_{v}, M_{u}$ are the operators of multiplication by $v, u$. In other words, the integral operator $K(\lambda)$ can be also written as $K(\lambda)=-\overline{M_{v}\left(H_{0}-\lambda\right)^{-1} M_{u}} \in \mathcal{B}_{2}\left(L^{2}(\mathbb{R} ; d \xi)^{n}\right)$. From now on, we will use the notation $K(\lambda)$ also for the operator $-\overline{M_{v}\left(H_{0}-\lambda\right)^{-1} M_{u}}$.

We introduce the following matrices by taking the Fourier transform in (3.24) and (3.25):

$$
\begin{gathered}
H_{0 \eta}=\left[\begin{array}{cc}
-\eta^{2} D_{\ell}+c \mu+F_{11}^{\prime}(Q(\infty)) & F_{12}^{\prime}(Q(\infty)) \\
F_{21}^{\prime}(Q(\infty)) & c i \eta+F_{22}^{\prime}(Q(\infty))
\end{array}\right], \eta \in \mathbb{R}, \\
T_{\eta}(\lambda)=\left[\begin{array}{ccc}
i \eta & 0 & -I \\
c^{-1} F_{21}^{\prime}(Q(\infty)) & c^{-1}\left(-\lambda+F_{22}^{\prime}(Q(\infty))\right)+i \eta & 0 \\
D_{\ell}^{-1}\left(-\lambda+F_{11}^{\prime}(Q(\infty))\right) & D_{\ell}^{-1} F_{12}^{\prime}(Q(\infty)) & c D_{\ell}^{-1}+i \eta
\end{array}\right] .
\end{gathered}
$$

Assume $\lambda \in \rho\left(H_{0}\right)$. Let us denote by $H_{i j}, \widetilde{H}_{i j}, \widehat{H}_{i j}$ the blocks of the block-operators $\left(H_{0 \eta}-\lambda\right)$, $\left(H_{0 \eta}-\lambda\right)^{-1},\left(H_{0}-\lambda\right)^{-1}$, respectively, in the direct sum decomposition $L^{2}(\mathbb{R} ; d \xi)^{n}=L^{2}(\mathbb{R} ; d \xi)^{\ell} \oplus$ $L^{2}(\mathbb{R} ; d \xi)^{n-\ell}$ such that

$$
\left(H_{0 \eta}-\lambda\right)=\left[H_{i j}\right]_{i, j=1}^{2},\left(H_{0 \eta}-\lambda\right)^{-1}=\left[\widetilde{H}_{i j}\right]_{i, j=1}^{2},\left(H_{0}-\lambda\right)^{-1}=\left[\widehat{H}_{i j}\right]_{i, j=1}^{2} .
$$

Lemma 35. The inverse of the operator $T(\lambda)$ from (3.24) is given by the formula

$$
T(\lambda)^{-1}=\left[\begin{array}{ccc}
\left(c+\partial_{\xi} D_{\ell}\right) \widehat{H}_{11} & c \widehat{H}_{12} & D_{\ell} \widehat{H}_{11} \\
\left(c+\partial_{\xi} D_{\ell}\right) \widehat{H}_{21} & c \widehat{H}_{22} & D_{\ell} \widehat{H}_{21} \\
-I+\partial_{\xi}\left(c+\partial_{\xi} D_{\ell}\right) \widehat{H}_{11} & c \partial_{\xi} \widehat{H}_{12} & \partial_{\xi} D_{\ell} \widehat{H}_{11}
\end{array}\right], \lambda \in \rho\left(H_{0}\right) .
$$

Proof. Since $\lambda \in \rho\left(H_{0}\right)$, the invertibility of the matrix $H_{0 \eta}-\lambda$ follows from Lemma 30. A direct verification shows that the inverse of the matrix $T_{\eta}(\lambda)$ is given by the formula

$$
T_{\eta}^{-1}(\lambda)=\left[\begin{array}{ccc}
\left(c+i \eta D_{\ell}\right) \widetilde{H}_{11} & c \widetilde{H}_{12} & D_{\ell} \widetilde{H}_{11} \\
\left(c+i \eta D_{\ell}\right) \widetilde{H}_{21} & c \widetilde{H}_{22} & D_{\ell} \widetilde{H}_{21} \\
-I+i \eta\left(c+i \eta D_{\ell}\right) \widetilde{H}_{11} & c i \eta \widetilde{H}_{12} & i \eta D_{\ell} \widetilde{H}_{11}
\end{array}\right]
$$

Taking the Fourier transform in (3.42) proves the required assertion (3.41).

Next, we consider the matrices $u(\xi)$ and $v(\xi)$ defined in (3.34). Using the block representation $u(\xi)=\left[\begin{array}{ll}u_{11} & u_{12} \\ u_{21} & u_{22}\end{array}\right], v(\xi)=\left[\begin{array}{ll}v_{11} & v_{12} \\ v_{21} & v_{22}\end{array}\right]$ in the direct sum decomposition $\mathbb{C}^{n}=\mathbb{C}^{\ell} \oplus \mathbb{C}^{n-\ell}$, we introduce the matrices $\widetilde{u}(\xi), \widetilde{v}(\xi)$ and the operator $\mathbb{K}(\lambda)$ as follows:

$$
\widetilde{u}(\xi)=\left[\begin{array}{ccc}
0_{\ell \times \ell} & 0_{\ell \times(n-\ell)} & 0_{\ell \times \ell} \\
c^{-1} u_{21} & c^{-1} u_{22} & 0_{(n-\ell) \times \ell} \\
D_{\ell}^{-1} u_{11} & D_{\ell}^{-1} u_{12} & 0_{\ell \times \ell}
\end{array}\right], \widetilde{v}(\xi)=\left[\begin{array}{ccc}
v_{11} & v_{12} & 0_{\ell \times \ell} \\
v_{21} & v_{22} & 0_{(n-\ell) \times \ell} \\
0_{\ell \times \ell} & 0_{\ell \times(n-\ell)} & 0_{\ell \times \ell}
\end{array}\right],
$$




$$
\mathbb{K}(\lambda)=-\overline{M_{\widetilde{v}} T(\lambda)^{-1} M_{\widetilde{u}}}, \quad \lambda \in \rho\left(H_{0}\right),
$$

where the operator $M_{\widetilde{v}} T(\lambda)^{-1} M_{\widetilde{u}}$ is originally defined on the (maximal) domain of $M_{\widetilde{u}}$. We will see in (3.47) that $\mathbb{K}(\lambda) \in \mathcal{B}\left(L^{2}(\mathbb{R} ; d \xi)^{n+\ell}\right)$. We remark that $B(\xi)$ in (3.28) can be written as $B(\xi)=\widetilde{u}(\xi) \widetilde{v}(\xi)$.

Hypothesis 36. We assume:

$$
\lambda_{0} \in \rho\left(H_{0}\right) \cap\{\lambda \in \mathbb{C} \mid \lambda \text { is an isolated eigenvalue of } H\} .
$$

Theorem 37. Assume Hypothesis 36 and let $\mathbb{E}(\cdot)$ be the Evans function for the perturbed equation (3.29). Then

$$
m\left(\lambda_{0} ; \mathbb{E}(\cdot)\right)=m\left(\lambda_{0} ; I_{L^{2}(\mathbb{R})^{n+\ell}}-\mathbb{K}(\cdot)\right)=m\left(\lambda_{0} ; I_{L^{2}(\mathbb{R})^{n}}-K(\cdot)\right)=m\left(\lambda_{0} ; H\right) .
$$

Proof. The first equality follows from formula (3.15). The second equality follows from the identity

$$
\mathbb{K}(\lambda)=\left[\begin{array}{cc}
K(\lambda) & 0_{n \times \ell} \\
0_{\ell \times n} & 0_{\ell \times \ell}
\end{array}\right], \lambda \in \rho\left(H_{0}\right),
$$

which, in turn, follows from (3.41) and (3.43):

$$
\begin{aligned}
& \mathbb{K}(\lambda)=-\overline{M_{\widetilde{v}} T(\lambda)^{-1} M_{\widetilde{u}}} \\
& =-\left[\begin{array}{cc}
{\left[\begin{array}{ll}
v_{11} & v_{12} \\
v_{21} & v_{22}
\end{array}\right]\left[\begin{array}{cc}
c \widehat{H}_{12} & D_{\ell} \widehat{H}_{11} \\
c \widehat{H}_{22} & D_{\ell} \widehat{H}_{21}
\end{array}\right]\left[\begin{array}{cc}
c^{-1} u_{21} & c^{-1} u_{22} \\
D_{\ell}^{-1} u_{11} & D_{\ell}^{-1} u_{12}
\end{array}\right]} & 0 \\
0 &
\end{array}\right] \\
& =-\left[\begin{array}{cc}
{\left[\begin{array}{cc}
v_{11} & v_{12} \\
v_{21} & v_{22}
\end{array}\right]\left[\begin{array}{cc}
\widehat{H}_{12} u_{21}+\widehat{H}_{11} u_{11} & \widehat{H}_{12} u_{22}+\widehat{H}_{11} u_{12} \\
\widehat{H}_{22} u_{21}+\widehat{H}_{21} u_{11} & \widehat{H}_{22} u_{22}+\widehat{H}_{21} u_{12}
\end{array}\right]} & 0 \\
0 &
\end{array}\right]
\end{aligned}
$$

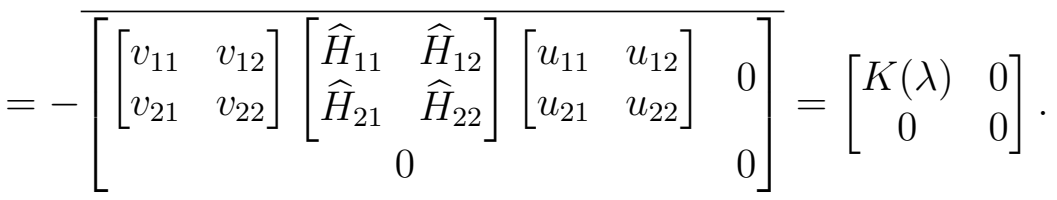

By Theorem 33, from (3.47) we derive $\mathbb{K}(\lambda) \in \mathcal{B}_{2}\left(L^{2}(\mathbb{R} ; d \xi)^{n+\ell}\right)$ and

$$
\operatorname{det}_{2}\left(I_{L^{2}(\mathbb{R} ; d \xi)^{n+\ell}}-\mathbb{K}(\lambda)\right)=\operatorname{det}_{2}\left(I_{L^{2}(\mathbb{R} ; d \xi)^{n}}-K(\lambda)\right),
$$

yielding $m\left(\lambda_{0} ; I_{L^{2}(\mathbb{R} ; d \xi)^{n+\ell}}-\mathbb{K}(\cdot)\right)=m\left(\lambda_{0} ; I_{L^{2}(\mathbb{R} ; d \xi)^{n}}-K(\cdot)\right)$. Finally, the last equality in (3.46) follows from Theorem 21 and Theorem 18. 


\subsection{General $n$-th Order Linear Differential Equations}

Let us consider the eigenvalue problem associated with the general $n$-th order linear differential operator:

$$
a_{n} \partial_{x}^{n} U(x)+\sum_{l=0}^{n-1} a_{l}(x) \partial_{x}^{l} U(x)=\lambda U(x), \quad x \in \mathbb{R},
$$

where $a_{n}$ is a non-zero constant and $a_{l}(\cdot), l=0, \ldots, n-1$, are given $L_{\text {loc }}^{1}(\mathbb{R})$-functions. Equation (3.49) is equivalent to the first order system:

$$
\Psi^{\prime}(x)=\left[\begin{array}{ccccc}
0 & 1 & \cdots & \cdots & 0 \\
0 & 0 & 1 & \cdots & 0 \\
\vdots & & & & \vdots \\
0 & & & & 1 \\
\frac{\lambda-a_{0}(x)}{a_{n}} & -\frac{a_{1}(x)}{a_{n}} & \cdots & \cdots & -\frac{a_{n-1}(x)}{a_{n}}
\end{array}\right] \Psi(x), \Psi=\left[\begin{array}{c}
U \\
U^{\prime} \\
\vdots \\
U^{(n-2)} \\
U^{(n-1)}
\end{array}\right]
$$

Hypothesis 38. Assume that the following limits exist:

$$
a_{l}^{\infty}:=\lim _{|x| \rightarrow \infty} a_{l}(x), l=0, \ldots, n-1 .
$$

Assume Hypothesis 38. Denoting by $W_{2}^{k}$ the Sobolev space of $k$ times differentiable functions with $L^{2}$-derivatives, let us introduce the operators $T(\lambda), H_{0}, H, K(\lambda)$, and the matrices $A, B$ as follows:

$$
T(\lambda)=\left[\begin{array}{ccccc}
\partial_{x} & -1 & \cdots & \cdots & 0 \\
0 & \partial_{x} & -1 & \cdots & 0 \\
\vdots & & & & \vdots \\
0 & & & \partial_{x} & -1 \\
\frac{a_{0}^{\infty}-\lambda}{a_{n}} & \frac{a_{1}^{\infty}}{a_{n}} & \cdots & \cdots & \partial_{x}+\frac{a_{n-1}^{\infty}}{a_{n}}
\end{array}\right]
$$

with the domain $\left(\operatorname{dom}(T(\lambda)),\|\cdot\|_{T(\lambda)}\right)=W_{2}^{1}(\mathbb{R})^{n}$,

$$
H_{0}=a_{n} \partial_{x}^{n}+\sum_{l=0}^{n-1} a_{l}^{\infty} \partial_{x}^{l}
$$

with the domain $\left(\operatorname{dom}\left(H_{0}\right),\|\cdot\|_{H_{0}}\right)=W_{2}^{n}(\mathbb{R})$,

$$
H=H_{0}+V, \text { where } V(x)=\sum_{l=0}^{n-1}\left(a_{l}(x)-a_{l}^{\infty}(x)\right) \partial_{x}^{l},
$$

with the domain $\left(\operatorname{dom}(H),\|\cdot\|_{H}\right)=W_{2}^{n}(\mathbb{R})$, and

$$
K(\lambda)=\left(H-H_{0}\right)\left(H_{0}-\lambda\right)^{-1}, \lambda \in \rho\left(H_{0}\right),
$$




$$
\begin{aligned}
& A(\lambda)=\left[\begin{array}{ccccc}
0 & 1 & \cdots & \cdots & 0 \\
0 & 0 & 1 & \cdots & 0 \\
\vdots & & & & \vdots \\
0 & & & & 1 \\
\frac{\lambda-a_{0}^{\infty}}{a_{n}} & -\frac{a_{1}^{\infty}}{a_{n}} & \cdots & \cdots & -\frac{a_{n-1}^{\infty}}{a_{n}}
\end{array}\right] \\
& B(x)=\left[\begin{array}{cccc}
0 & 0 & \cdots & 0 \\
0 & 0 & \cdots & 0 \\
\vdots & & & \vdots \\
\frac{-a_{0}(x)+a_{0}^{\infty}}{a_{n}} & \frac{-a_{1}(x)+a_{1}^{\infty}}{a_{n}} & \cdots & \frac{-a_{n-1}(x)+a_{n-1}^{\infty}}{a_{n}}
\end{array}\right] \text {. }
\end{aligned}
$$

Then the eigenvalue problem (3.50) can be recast as

$$
\Psi^{\prime}(x)=(A(\lambda)+B(x)) \Psi(x) .
$$

Preparing to use the Fourier transform, we introduce the following matrices obtained by replacing $\partial_{x}$ by $i \eta$ in (3.53) and (3.52):

$$
\begin{gathered}
H_{0 \eta}=a_{n}(i \eta)^{n}+\sum_{l=0}^{n-1} a_{l}^{\infty}(i \eta)^{l}, \eta \in \mathbb{R}, \\
T_{\eta}(\lambda)=\left[\begin{array}{ccccc}
i \eta & -1 & \cdots & \cdots & 0 \\
0 & i \eta & -1 & \cdots & 0 \\
\vdots & & & & \vdots \\
0 & & & i \eta & -1 \\
\frac{a_{0}^{\infty}-\lambda}{a_{n}} & \frac{a_{1}^{\infty}}{a_{n}} & \cdots & \cdots & i \eta+\frac{a_{n-1}^{\infty}}{a_{n}}
\end{array}\right] .
\end{gathered}
$$

Lemma 39. The inverse of the operator $T(\lambda)$ is given by the formula

$$
T(\lambda)^{-1}=\left[\begin{array}{cccc}
* & \cdots & * & a_{n}\left(H_{0}-\lambda\right)^{-1} \\
* & \cdots & * & a_{n} \partial_{x}\left(H_{0}-\lambda\right)^{-1} \\
\vdots & & \vdots & \vdots \\
* & \cdots & * & a_{n} \partial_{x}^{(n-1)}\left(H_{0}-\lambda\right)^{-1}
\end{array}\right], \lambda \in \rho\left(H_{0}\right),
$$

where stars denote the elements which are not important in the sequel.

Proof. Since $\lambda \in \rho\left(H_{0}\right)$, the matrix $H_{0 \eta}-\lambda$ is invertible. A direct calculation shows that the inverse of the matrix $T_{\eta}(\lambda)$ is given by the formula

$$
T_{\eta}(\lambda)^{-1}=\left[\begin{array}{cccc}
* & \cdots & * & a_{n}\left(H_{0 \eta}-\lambda\right)^{-1} \\
* & \cdots & * & a_{n}(i \eta)\left(H_{0 \eta}-\lambda\right)^{-1} \\
\vdots & & \vdots & \vdots \\
* & \cdots & * & a_{n}(i \eta)^{(n-1)}\left(H_{0 \eta}-\lambda\right)^{-1}
\end{array}\right] .
$$


Using the Fourier transform in (3.61) proves the required assertion (3.60).

Next, we introduce the operator $\mathbb{K}(\lambda)$ on $L^{2}(\mathbb{R} ; d x)^{n}$ as follows:

$$
\mathbb{K}(\lambda)=-B(\cdot) T(\lambda)^{-1}, \quad \lambda \in \rho\left(H_{0}\right) .
$$

We will see in Theorem 41 that $\mathbb{K}(\lambda) \in \mathcal{B}\left(L^{2}(\mathbb{R} ; d x)^{n}\right)$.

Hypothesis 40. In addition to Hypothesis 38 , we assume that $a_{l}(\cdot)-a_{l}^{\infty} \in L^{2}(\mathbb{R}), l=0, \ldots, n-1$.

Theorem 41. Assume Hypothesis 40 and let $\lambda \in \rho\left(H_{0}\right)$. Then $\mathbb{K}(\lambda) \in \mathcal{B}_{2}\left(L^{2}(\mathbb{R} ; d x)^{n}\right)$.

Proof. The assertion in the theorem now follows from the well-known formula for the $\mathcal{B}_{2}$-norm of an integral operator, see [2, Theorem 11.3.6]:

$$
\begin{aligned}
& \|\mathbb{K}(\lambda)\|_{\mathcal{B}_{2}\left(L^{2}(\mathbb{R} ; d x)^{n}\right)}^{2}=\iint_{\mathbb{R} \times \mathbb{R}} d x d x^{\prime}\left\|\mathbb{K}\left(\lambda, x, x^{\prime}\right)\right\|_{\mathbb{C}^{n \times n}}^{2} \\
& \quad=\iint_{\mathbb{R} \times \mathbb{R}} d x d x^{\prime}\left\|-B(x)\left(T_{\eta}^{-1}\right)^{\vee}\left(\lambda, x-x^{\prime}\right)\right\|_{\mathbb{C}^{n \times n}}^{2} \\
& \quad \leq \int_{\mathbb{R}} d x\|B(x)\|_{\mathbb{C}^{n \times n}}^{2} \int_{\mathbb{R}} d x\left\|\left(T_{\eta}^{-1}\right)^{\vee}\left(\lambda, x-x^{\prime}\right)\right\|_{\mathbb{C}^{n \times n}}^{2}=\|B\|_{L^{2}}^{2}\left\|T_{\eta}(\lambda)^{-1}\right\|_{L^{2}}^{2}<\infty .
\end{aligned}
$$

The last inequality holds since $\|B\|_{L^{2}}^{2}<\infty$ by Hypothesis 40 , and since the $L^{2}$-norm of each entry of the matrix $T_{\eta}(\lambda)^{-1}$ is finite.

Hypothesis 42. We assume:

$$
\lambda_{0} \in \rho\left(H_{0}\right) \cap\{\lambda \in \mathbb{C} \mid \lambda \text { is an isolated eigenvalue of } H\} .
$$

Theorem 43. Assume Hypothesis 42 and let $\mathbb{E}(\cdot)$ be the Evans function for the perturbed equation (3.58). Then

$$
m\left(\lambda_{0} ; \mathbb{E}(\cdot)\right)=m\left(\lambda_{0} ; I_{L^{2}(\mathbb{R})^{n}}-\mathbb{K}(\cdot)\right)=m\left(\lambda_{0} ; I_{L^{2}(\mathbb{R})}-K(\cdot)\right)=m\left(\lambda_{0} ; H\right) .
$$

Proof. The first equality follows from formula (3.15). The second equality is based on the identity

$$
\mathbb{K}(\lambda)=\left[\begin{array}{cc}
0 & 0 \\
* & \left(H-H_{0}\right)\left(H_{0}-\lambda\right)^{-1}
\end{array}\right],
$$

which, in turn, follows from (3.57) and (3.60):

$$
\mathbb{K}(\lambda)=-B(x) T(\lambda)^{-1}
$$




$$
\begin{aligned}
& =-\left[\begin{array}{cccc}
0 & 0 & \cdots & 0 \\
0 & 0 & \cdots & 0 \\
\vdots & & & \vdots \\
\frac{-a_{0}+a_{0}^{\infty}}{a_{n}} & \frac{-a_{1}+a_{1}^{\infty}}{a_{n}} & \cdots & \frac{-a_{n-1}+a_{n-1}^{\infty}}{a_{n}}
\end{array}\right]\left[\begin{array}{cccc}
* & \cdots & * & a_{n}\left(H_{0}-\lambda\right)^{-1} \\
* & \cdots & * & a_{n} \partial_{x}\left(H_{0}-\lambda\right)^{-1} \\
\vdots & & \vdots & \vdots \\
* & \cdots & * & a_{n} \partial_{x}^{(n-1)}\left(H_{0}-\lambda\right)^{-1}
\end{array}\right] \\
& =\left[\begin{array}{cc}
0 & 0 \\
* & \left(H-H_{0}\right)\left(H_{0}-\lambda\right)^{-1}
\end{array}\right] .
\end{aligned}
$$

By Theorem 41, from (3.65) we derive

$$
\operatorname{det}_{2}\left(I_{L^{2}(\mathbb{R} ; d x)^{n}}-\mathbb{K}(\lambda)\right)=\operatorname{det}_{2}\left(I_{L^{2}(\mathbb{R} ; d x)}-\left(H-H_{0}\right)\left(H_{0}-\lambda\right)^{-1}\right),
$$

yielding the second equality in (3.64). Finally, the last equality in (3.64) follows from Theorem 21 and Theorem 18.

\section{Acknowledgements}

This work was partially supported by the US National Science Foundation under Grant NSF DMS0754705, by the Research Board and Research Council of the University of Missouri. Our special thanks go to Konstantin A. Makarov for suggesting identity (3.12), which is one of the main ingredients of this work. Also, we thank Fritz Gesztesy for help and many suggestions and discussions.

\section{References}

[1] J. Alexander, R. Gardner, C. Jones. A topological invariant arising in the stability analysis of traveling waves. J. reine angew. Math., 410 (1990), 167-212.

[2] M. S. Birman, M. Z. Solomyak. Spectral theory of self-adjoint operators in Hilbert space. Reidel, Dordrecht, 1987.

[3] C. Chicone, Y. Latushkin. Evolution semigroups in dynamical systems and differential equations. Amer. Math. Soc., Providence, RI, 1999.

[4] R. A. Gardner, C. K. R. T. Jones. Traveling waves of a perturbed diffusion equation arising in a phase field model. Indiana Univ. Math. J., 39 (1989), 1197-1222.

[5] F. Gesztesy, Y. Latushkin, K. A. Makarov. Evans functions, Jost functions, and Fredholm determinants. Arch. Rat. Mech. Anal., 186 (2007), 361-421.

[6] F. Gesztesy, Y. Latushkin, M. Mitrea, M. Zinchenko. Non-self-adjoint operators, infinite determinants, and some applications. Russ. J. Math. Phys., 12 (2005), 443-471.

[7] F. Gesztesy, Y. Latushkin, K. Zumbrun. Derivatives of (modified) Fredholm determinants and stability of standing and traveling waves. J. Math. Pures Appl., 90 (2008), 160-200. 
[8] F. Gesztesy, K. A. Makarov. (Modified) Fredholm determinants for operators with matrixvalued semi-separable integral kernels revisited. Integral Eq. Operator Theory, 47 (2003), 457-497; Erratum. 48 (2004), 425-426.

[9] I. Gohberg, S. Goldberg, M. Kaashoek. Classes of linear operators. Vol. 1. Birkhäuser, 1990.

[10] K. F. Gurski, R. Kollar, R. L. Pego. Slow damping of internal waves in a stably stratified fluid. Proc. Royal Soc. Lond. Ser. A Math. Phys. Engrg. Sci., 460 (2004), 977-994.

[11] K. F. Gurski, R. L. Pego. Normal modes for a stratified viscous fluid layer. Proc. Royal Soc. Edinburgh Sect. A, 132 (2002), 611-625.

[12] T. Kapitula, B. Sandstede. Edge bifurcations for near integrable systems via Evans function techniques. SIAM J. Math. Anal., 33 (2002), 1117-1143.

[13] T. Kapitula, B. Sandstede. Eigenvalues and resonances using the Evans function. Discrete Contin. Dyn. Syst., 10 (2004), 857-869.

[14] T. Kato. Wave operators and similarity for some non-selfadjoint operators. Math. Ann., 162 (1966), 258-279.

[15] R. L. Pego, M. I. Weinstein. Eigenvalues and instabilities of solitary waves. Philos. Trans. Royal Soc. London Ser. A, 340 (1992), 47-94.

[16] M. Reed, B. Simon. Methods of modern mathematical physics. I: Functional analysis. Academic Press, New York, 1980.

[17] M. Reed, B. Simon. Methods of Modern Mathematical Physics. II: Fourier Analysis, Selfadjointness. Academic Press, New York, 1975.

[18] B. Sandstede. Stability of traveling waves. In: Handbook of dynamical systems. Vol. 2. B. Hasselblatt, A. Katok (eds.). North-Holland, Elsevier, Amsterdam, 2002, pp. 983-1055.

[19] B. Simon. Trace ideals and their applications. Cambridge University Press, Cambridge, 1979.

[20] K. Zumbrun. Multidimensional stability of planar viscous shock waves. In: Advances in the Theory of Shock Waves. T.-P. Liu, H. Freistühler, A. Szepessy (eds.). Progress Nonlin. Diff. Eqs. Appls., 47, Birkhäuser, Boston, 2001, pp. 307-516. 